\title{
Direct and efficient cellular transformation of primary rat mesenchymal precursor cells by KSHV
}

\author{
Tiffany Jones, 1,2,3 Fengchun Ye,1,4 Roble Bedolla, ${ }^{1,4}$ Yufei Huang, ${ }^{1,5}$ \\ Jia Meng, 5 Liwu Qian,1,4 Hongyi Pan,1,4 Fuchun Zhou,,1,4 \\ Rosalie Moody,, ${ }^{1,4}$ Brent Wagner, 6 Mazen Arar, ${ }^{4}$ and Shou-Jiang Gao 1,2,3,4,6 \\ ${ }^{1} T u m o r$ Virology Program, Greehey Children's Cancer Research Institute, ${ }^{2}$ Department of Molecular Medicine, \\ University of Texas Health Science Center at San Antonio, San Antonio, Texas, USA. \\ ${ }^{3}$ Department of Molecular Microbioloby and Immunology, Keck School of Medicine, University of Southern California, Los Angeles, California, USA. \\ 4Department of Pediatrics, University of Texas Health Science Center at San Antonio, San Antonio, Texas, USA. \\ ${ }^{5}$ Department of Electrical and Computer Engineering, University of Texas at San Antonio, San Antonio, Texas, USA. \\ ${ }^{6}$ Department of Medicine, University of Texas Health Science Center at San Antonio, San Antonio, Texas, USA.
}

\begin{abstract}
Infections by viruses are associated with approximately $12 \%$ of human cancer. Kaposi's sarcoma-associated herpesvirus (KSHV) is causally linked to several malignancies commonly found in AIDS patients. The mechanism of KSHV-induced oncogenesis remains elusive, due in part to the lack of an adequate experimental system for cellular transformation of primary cells. Here, we report efficient infection and cellular transformation of primary rat embryonic metanephric mesenchymal precursor cells (MM cells) by KSHV. Cellular transformation occurred at as early as day 4 after infection and in nearly all infected cells. Transformed cells expressed hallmark vascular endothelial, lymphatic endothelial, and mesenchymal markers and efficiently induced tumors in nude mice. KSHV established latent infection in MM cells, and lytic induction resulted in low levels of detectable infectious virions despite robust expression of lytic genes. Most KSHV-induced tumor cells were in a latent state, although a few showed heterogeneous expression of lytic genes. This efficient system for KSHV cellular transformation of primary cells might facilitate the study of growth deregulation mechanisms resulting from KSHV infections.
\end{abstract}

\section{Introduction}

Oncogenic viruses are associated with close to $12 \%$ of human cancer (1). Kaposi's sarcoma-associated herpesvirus (KSHV) is causally linked to Kaposi's sarcoma (KS), primary effusion lymphoma, and a subset of multicentric Castleman's disease, malignancies commonly found in AIDS patients (2). While KSHV encodes an array of genes targeting cellular oncogenic, tumor suppressor, and survival pathways, the mechanism of KSHV-induced oncogenesis remains unclear, in part because of the lack of an efficient system for cellular transformation of primary cells.

KSHV-infected tumor cells manifest the hallmark spindle-shape morphology, expressing vascular endothelial, lymphatic endothelial and mesenchymal markers (2). In culture, KSHV infects a variety of cell types. KSHV infection of human vascular and lymphatic endothelial cells converts them into spindle-shaped cells expressing both lineage markers (3-11). Although the lifespan of primary human endothelial cells is prolonged by KSHV, their continuous growth relies on exogenous growth factors, and cellular transformation remains elusive (3-5). These KSHV-infected cells do not induce any tumors when inoculated into immunocompromised mice.

The initial KSHV target cells could be precursor cells, and KSlike spindle endothelial precursor cells are present in the blood of KS patients $(12,13)$. Indeed, KSHV infects human mesenchymal

Authorship note: Tiffany Jones, Fengchun Ye, Roble Bedolla, and Shou-Jiang Gao contributed equally to this work.

Conflict of interest: The authors have declared that no conflict of interest exists. Citation for this article: J Clin Invest. 2012;122(3):1076-1081. doi:10.1172/JCI58530 stem cells and hematopoietic precursor cells $(14,15)$. However, KSHV fails to immortalize and transform these cells. In contrast, transfection of mouse liver precursor cells with recombinant KSHV BAC36 genomes resulted in immortalization of a subset of cells, which induced tumors in nude mice despite the lack of cellular transformation phenotypes in culture (16). Nevertheless, KSHV infection as well as transfection and maintenance of KSHV genomes in mouse cells are inefficient. Here, we show that KSHV efficiently infects and transforms primary rat embryonic metanephric mesenchymal precursor (MM) cells. KSHV-transformed MM (KMM) cells efficiently induce tumors in nude mice. These results should facilitate study of the mechanisms of growth deregulation by KSHV infection.

\section{Results and Discussion}

$K S H V$ efficiently infects MM cells. To identify primary cell type(s) that can be transformed by KSHV, we infected cells of different origins (Table 1). MM cells were efficiently infected and transformed by KSHV. KSHV infection was monitored by examining the expression of a GFP cassette in viral genome (ref. 17 and Figure 1A). KMM cells were positive for latent nuclear antigen (LANA, ORF73), with less than $1 \%$ positive for the late lytic protein ORF65 (Figure 1, B and C). Continuous passage of KMM cells changed neither the genome number per cell (Supplemental Figure 1; supplemental material available online with this article; doi:10.1172/ JCI58530DS1) nor the expression patterns of LANA and ORF65. Induction of viral lytic replication with 12-O-tetradecanoylphorbol-13-acetate (TPA) did not affect LANA expression but increased 
Table 1

KSHV infection of human, mouse, and rat cells

\begin{tabular}{|c|c|c|c|c|c|c|}
\hline Cells & Host & Cell type & $\begin{array}{l}\text { Infection } \\
\text { efficiency }\end{array}$ & $\begin{array}{l}\text { Persistence } \\
\text { efficiency }\end{array}$ & Immortalization & Transformation \\
\hline HUVECs & Human & Umbilical vein endothelial cells & High & High & Senescence & $\mathrm{N} / \mathrm{A}$ \\
\hline DMVECs & Human & Vascular endothelial cells & Medium & High & Senescence & $\mathrm{N} / \mathrm{A}$ \\
\hline MS1 cells & Mouse & Vascular endothelial cells & Low & Low & $\mathrm{N} / \mathrm{A}$ & $\mathrm{N} / \mathrm{A}$ \\
\hline SVEC cells & Mouse & Lymphatic endothelial cells & Low & Low & $\mathrm{N} / \mathrm{A}$ & $\mathrm{N} / \mathrm{A}$ \\
\hline MEFs & Mouse & Embryonic fibroblasts & High & Low & Senescence & $\mathrm{N} / \mathrm{A}$ \\
\hline JWEK cells & Mouse & Mesenchymal precursor cells & Low & Low & $\mathrm{N} / \mathrm{A}$ & $\mathrm{N} / \mathrm{A}$ \\
\hline RAOECs & Rat & Aortic endothelial cells & Low & Low & $\mathrm{N} / \mathrm{A}$ & $\mathrm{N} / \mathrm{A}$ \\
\hline MM cells & Rat & Mesenchymal precursor cells & High & High & Yes & Yes \\
\hline
\end{tabular}

NA, not applicable; MEF, mouse embryonic fibroblast; RAOEC, rat aortic endothelial cell.

ORF65-positive cells to $4 \%-5 \%$ (Figure $1, \mathrm{~B}$ and C). As controls, uninduced and induced MM cells were negative for LANA and ORF65 proteins (Supplemental Figure 2). The expression of lytic transcripts including transcripts of the immediate early gene RTA (ORF50), early genes ORF57, ORF59, kbZip (ORF-K8), vIL-6 (ORF-K2), and ORF49, and late genes ORF65 and ORF-K8.1 was increased, while that of the latent transcript $v$ Cyclin (ORF72) was not altered following lytic induction (Supplemental Figure 3). Interestingly, we detected only intermittent low levels of infectious virions in the supernatants of induced cells, suggesting an abortive viral lytic program. Similar results were observed when long-term-passage KMM cells were examined.
A

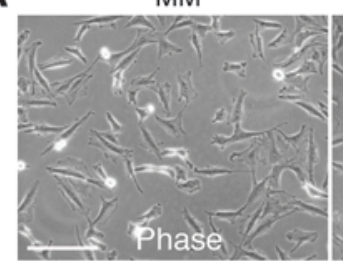

B

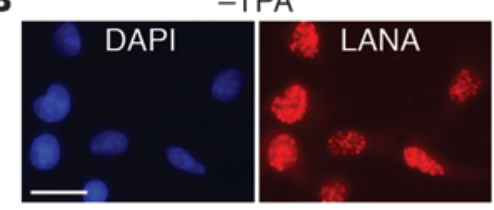

C

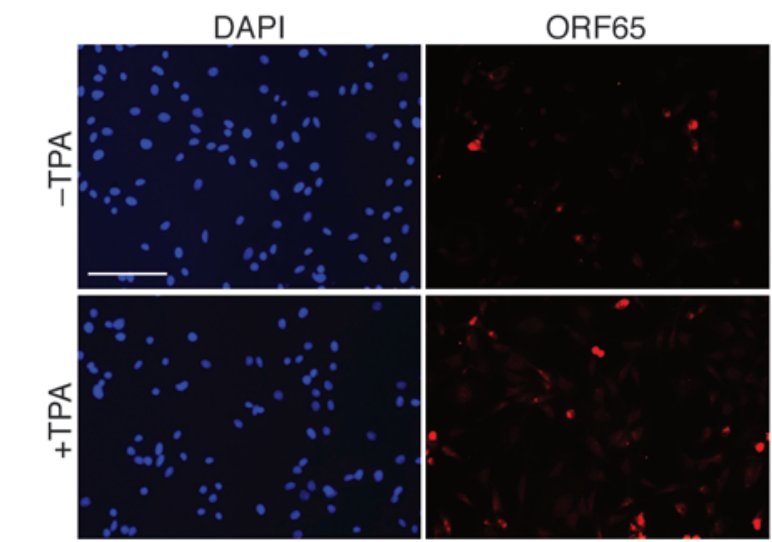

KMM
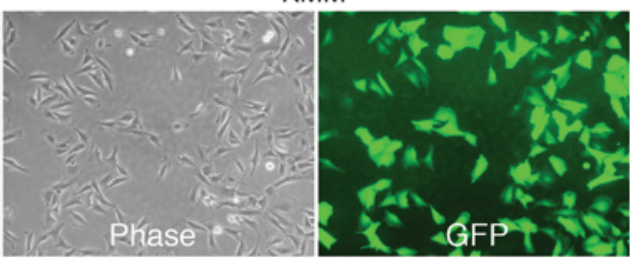

+ TPA

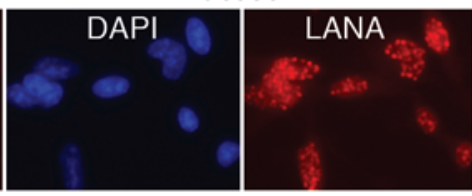

ORF65
D
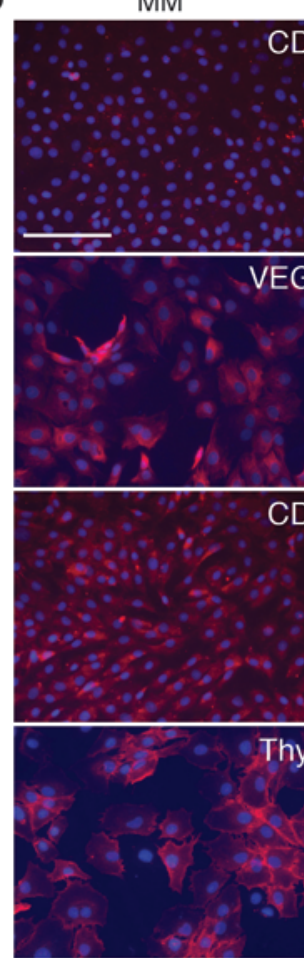

hy 1.1
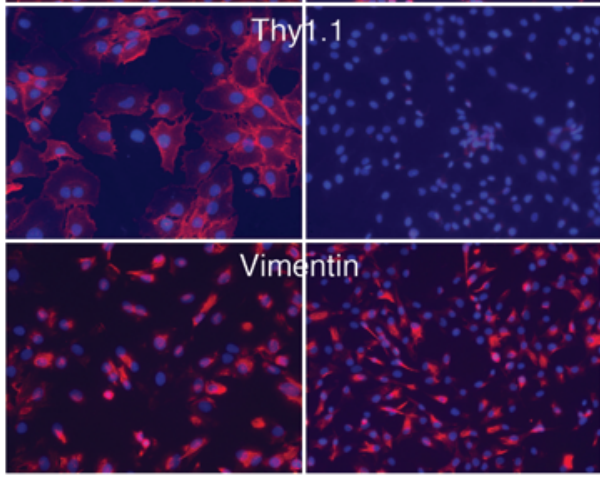

Figure 1

Characterization of MM and KMM cells. (A) Morphology at day 2 after seeding. Cells were seeded at $5 \times 10^{4}$ cells/well in 6 -well plates. (B and C) Expression of LANA (B) and ORF65 (C) proteins in uninduced KMM cells and KMM cells induced with TPA for 48 hours. (D) Expression of cell surface markers in MM and KMM cells. Scale bars: $50 \mu \mathrm{m}$ (A, C, and D), $10 \mu \mathrm{m}$ (B). 
A
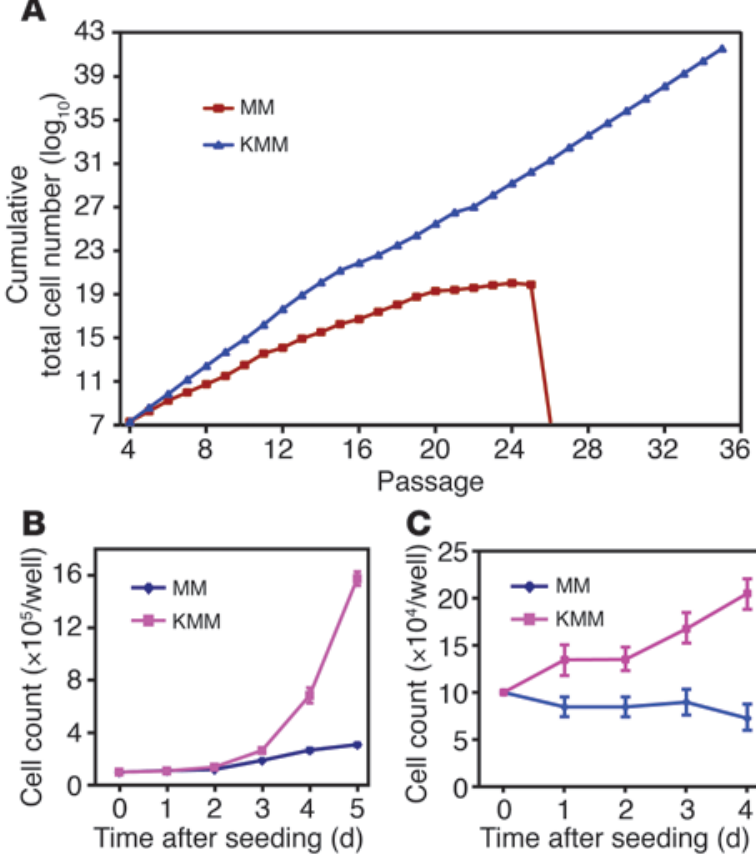

D
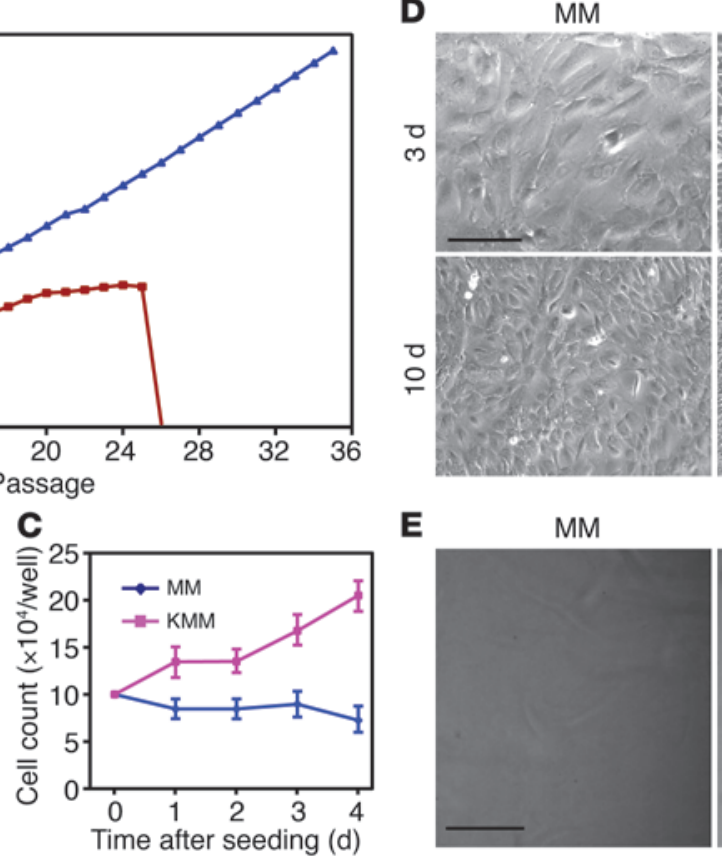

$\mathbf{E}$

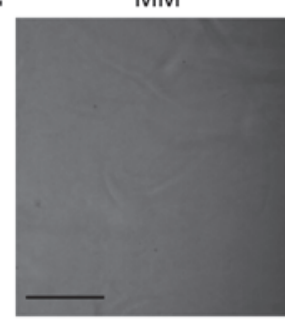

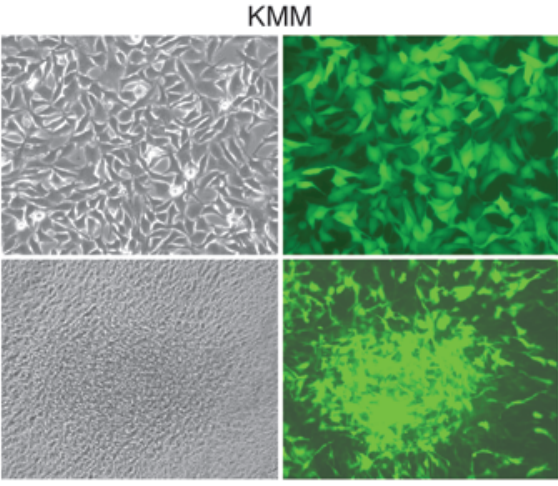

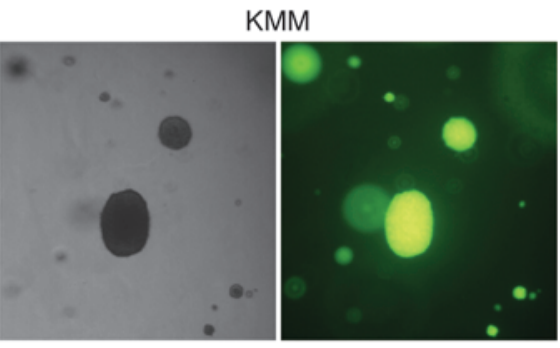

Figure 2

Immortalization and transformation of MM cells by KSHV. (A) MM cells underwent crisis after 25-28 passages. KMM grew continuously without crisis. Cells were passaged every 3 days at $2 \times 10^{4}$ cells/well in 24 -well plates. (B) KMM cells grew faster than MM cells in regular medium containing serum. Cells seeded at $10^{5}$ cells/well in 6-well plates were counted daily. (C) In serum-free medium, growth of MM cells stopped, while KMM cells continued to grow for up to day 4 after seeding. Cells seeded at $10^{5}$ cells/well in 6 -well plates were counted daily. (D) KMM cells formed foci, while MM cells were contact inhibited when they reached confluency. Cells seeded at $2 \times 10^{5}$ cells/well in 6-well plates were cultured with daily medium change. Scale bar: $40 \mu \mathrm{m}$. (E) KMM cells formed many large colonies in semisolid soft agar, while MM cells did not. Scale bar: $200 \mu \mathrm{m}$.

MM and KMM cells expressed vascular endothelial markers $\beta$-catenin, vWF, and VEGFR-1; lymphatic endothelial markers VEGFR-3 and LYVE-1; the mesenchymal marker vimentin; and the hematopoietic precursor marker CD34 (Figure 1D and Supplemental Figure 4). Vascular endothelial markers CD31, VCAM-1, and ICAM-1 and the lymphatic endothelial cell marker podoplanin were upregulated, while the hematopoietic precursor marker Thy1.1 was downregulated in KMM cells. Long-term passage of KMM cells did not change the patterns of cell surface markers. Thus, similar to vascular and lymphatic endothelial cells $(7,8,10)$, KSHV reprograms MM cells to express a mixture of vascular endothelial, lymphatic endothelial, mesenchymal, and hematopoietic precursor markers.

KMM cells are transformed. MM cells underwent crisis after 25-28 passages. In contrast, KMM cells were immortalized, as they could be maintained in continuous passage (Figure 2A). Compared with MM cells, KMM cells were smaller in size (Figure 1A) and had shorter doubling time as indicated by faster dilution of CFSE $(13.1 \pm 2.2$ vs. $19.4 \pm 2.4$ hours at exponential phase; Supplemental Figure 5A), which was also demonstrated by an increase in cells per cluster at day 2 after seeding (7-10 vs. 2-3 cells/cluster; Figure 1A) and a faster growth rate (Figure 2B). Accordingly, KMM cells had more cells in S phase (50\% vs. $40 \%)$ and fewer cells in $\mathrm{G}_{0} / \mathrm{G}_{1}$ phase (35\% vs. $42 \%$ ) than MM cells (Supplemental Figure $5 \mathrm{~B}$ ), which was due to accelerated transition from $G_{0} / G_{1}$ to $S$ phase, as shown by faster incorporation of BrdU (Supplemental Figure 5C). In serum-free medium, MM cells were growth arrested starting at day 1 after seeding, with $85 \%$ cells in $G_{0} / G_{1}$ phase and only $5 \%$ cells in S phase while KMM cells continued to proliferate for up to day 4 after seeding, with only $68 \%$ cells in $\mathrm{G}_{0} / \mathrm{G}_{1}$ phase and $20 \%$ in $\mathrm{S}$ phase, and continued to incorporate $\mathrm{BrdU}$ at a faster rate (Figure $2 \mathrm{C}$ and Supplemental Figure 5, D and E). MM cells were contact inhibited, while KMM cells lost contact inhibition, as shown by formation of foci and higher saturation density in culture (Figure 2D and Supplemental Figure 5F) and formation of colonies in semisolid soft agar (Figure 2E). Growth in semisolid soft agar was observed as early as day 4 after infection, the earliest time point examined, and with close to $100 \%$ efficiencies. These results indicated that MM cells were efficiently immortalized and transformed by KSHV. We reproduced these results 8 times with MM cells isolated from 3 different batches. This transformation phenotype did not change following long-term passage of KMM cells. Furthermore, virions from BCP-1 cells also efficiently infected and transformed MM cells (Supplemental Figure 6), indicating that the transformation phenotype was not restricted to a specific viral isolate.

KMM cells induce tumors in nude mice. While MM cells failed to induce any tumors in nude mice, KMM cells efficiently induced tumors, with a mean incidence of $84.6 \% \pm 11.2 \%$ and a mean latency of $12 \pm 1.6$ weeks (Figure 3, A and B). Tumors often manifested as reddish lesions and dissemination to visceral organs (Figure 3, C and D).

H\&E staining showed spindle-shaped tumor cells, many of which had mitotic figures and were positive for the proliferation marker Ki67 (Figure 3E and Supplemental Figure 7). Tumors contained many microvessels, slit-like spaces, and infiltrates of 
A

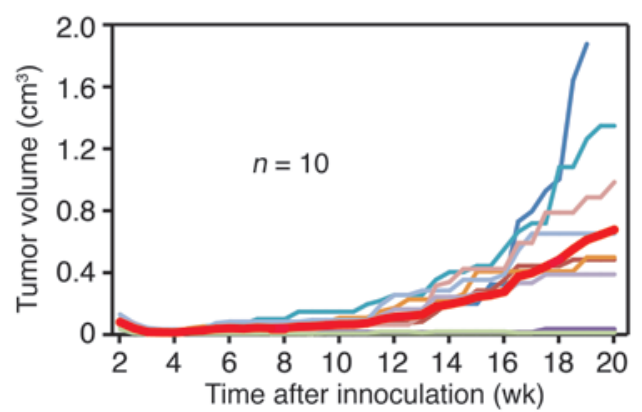

C

B

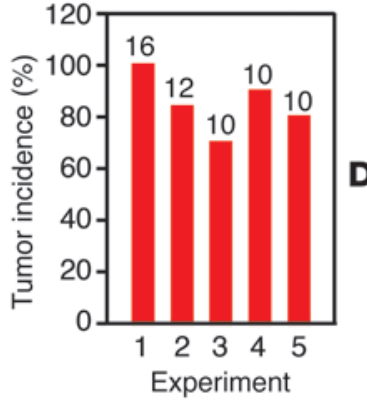

D
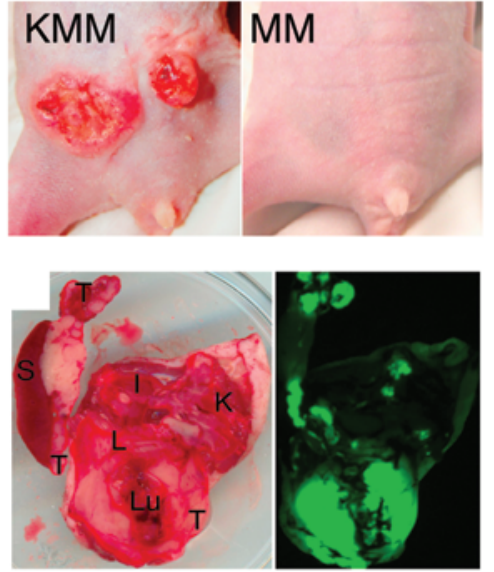

$\mathbf{E}$

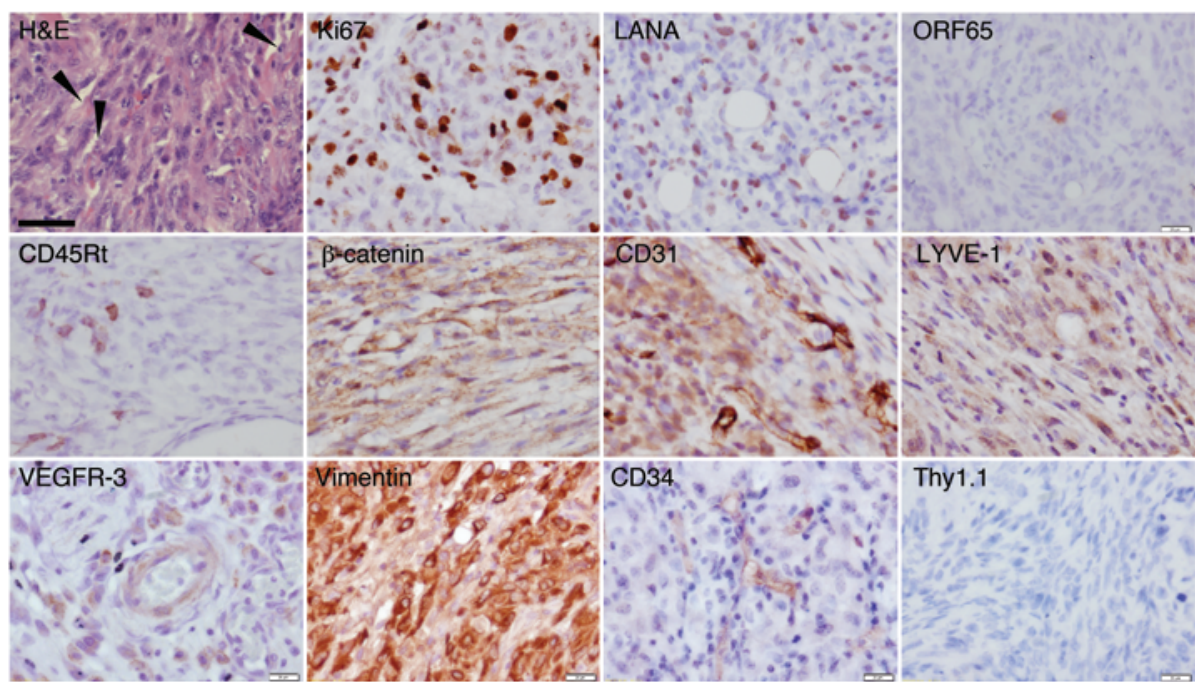

\section{Figure 3}

KMM cells induce tumors in nude mice. (A) Tumor growth curves. The red thick line indicates average tumor volumes. MM cells did not induce any tumors. (B) Tumor incidence from 5 independent experiments. Numbers above of the bars represent inoculated sites. Results in A were from experiment 3. (C) Representative reddish tumor morphology. (D) Tumor dissemination to visceral organs. Tumors (T) shown in green fluorescence were present in spleen (S), liver (L), lung (Lu), kidney (K), and intestine (I). (E) Immunohistochemical staining for H\&E, Ki67, LANA, and ORF65 proteins, the mouse B cell marker CD45Rt, vascular endothelial markers ( $\beta$-catenin and CD31), lymphatic endothelial markers (LYVE-1 and VEGFR-3), hematopoietic precursor markers (CD34 and Thy1.1), and the mesenchymal marker vimentin. Representative slit-like spaces are labeled with arrowheads. Scale bar: $40 \mu \mathrm{m}$.

immune cells such as $\mathrm{CD}_{4} 5 \mathrm{R}^{+}$mouse $\mathrm{B}$ cells, which were reminiscent of $\mathrm{CD}^{+} \mathrm{T}$ cells and CD20 $0^{+} \mathrm{B}$ cells in KS tumors (Supplemental Figure 8). These results indicated that KMM tumors consisted of proliferating spindle cells with vast inflammatory infiltrates and neoangiogenesis.

All tumor cells strongly expressed LANA protein, with $1 \%-3 \%$ of them expressing ORF65 protein (Figure 3E). Tumors had robust expression of latent LANA and $v$ Cyclin transcripts but heterogeneous expression of lytic transcripts (Supplemental Figure 9). RTA was at levels close to those in uninduced KMM cells in 2 of 14 tumors; ORF57 and ORF59 were at levels higher than in uninduced cells in 12 of 14 tumors; and ORF65 was detected in 5 of 14 tumors, of which only 1 was at a high level while the other 4 were at levels similar to those in uninduced KMM cells. These results indicated a spontaneous default lytic program in tumors.
KMM tumor cells were positive for vascular endothelial markers $\beta$-catenin, CD31, VE-cadherin, ICAM-1, and VCAM- 1 ; lymphatic endothelial markers LYVE-1, VEGFR-3, podoplanin, and PROX-1; hematopoietic precursor marker CD34; and mesenchymal marker vimentin, but were negative for Thy1.1 (Figure 3E and Supplemental Figure 7).

We have shown that KSHV can efficiently infect and transform MM cells. KMM cells were immortalized, proliferated at a faster rate than MM cells, grew in serum-free medium, lost contact inhibition in culture, and induced tumors in nude mice. Tumor cells acquired spindle morphology; formed slit-like spaces; and expressed vascular endothelial, lymphatic endothelial, and mesenchymal markers. Most tumor cells were in the latent state, but a few had heterogeneous expression of lytic genes. These pathological and viral features resemble those in KS tumors (Supple- 
mental Figure 8). This system should be useful for dissecting factors mediating KSHV-induced malignant transformation.

Because KSHV is a human virus, it is important to recognize the limitations of cross-species observations. While KSHV infects a variety of human cells in culture, cellular transformation of primary human cells remains challenging (2). Furthermore, explant tumor cells from most KS tumors are neither immortalized nor transformed, despite their in vivo malignant proliferative nature (18-21). While the loss of viral genome could account for the lack of cellular transformation, it is clear that MM cells are distinct from human primary cells.

Besides the mesenchymal marker vimentin, MM cells expressed vascular endothelial markers $\beta$-catenin, ICAM-1, vWF, and VEGFR-1; lymphatic endothelial markers VEGFR-3 and LYVE-1; and hematopoietic precursor markers CD34 and Thy1.1. KSHV infection upregulated the vascular endothelial markers VCAM-1 and CD31 and the lymphatic endothelial marker podoplanin. Interestingly, Thy1.1 expression was almost completely shut down, while that of CD34 was maintained following KSHV infection. In differentiated cells, CD34 is often expressed in endothelial cells, while Thy1.1 is more confined to hematopoietic cells $(22,23)$. Human Thy1 expression is associated with tumor suppression of ovarian cancer (24). Thus, KSHV appeared to downregulate Thy1.1 expression to inhibit its tumor-suppressive effect, while at the same time converting the mesenchymal precursor cells into a mixed phenotype of vascular and lymphatic endothelial cells. This reprogramming process is not surprising, because MM cells are multipotent and can be differentiated into diverse cell types, including endothelial cells $(25,26)$. Nevertheless, whether mesenchymal precursor cells are the KSHV targets in vivo requires further investigation.

\section{Methods}

Cell culture and virus infection. Primary HUVECs and microvascular endothelial cells (DMVECs) from Lonza, MS1 and SVEC cells from ATCC, and primary rat aortic endothelial cells from Lifeline Cell Technology were maintained as described by the vendors. Mouse and rat MM cells as well as embryonic fibroblasts were isolated and maintained as previously described $(27,28)$. Primary cells of fewer than 5 passages were used for infection experiments. For subsequent characterizations, MM and KMM cells between 5 and 10 passages were used. KMM cells were cultured with $150 \mu \mathrm{g} / \mathrm{ml}$ hygromycin, which was removed 1 week before experiments. Growth of BAC36 and BCP-1 KSHV and infection were performed as previously described $(5,17)$. Cells were split every 3 days, except for focus forma-

tion experiments, with daily change of medium. Cell numbers were determined at passage. Cells were observed daily for crisis, including growth arrest and cell death. Semisolid soft agar assay was performed as previously described (29). Cells at specific days following KSHV infection were seeded, and pictures of colonies were taken at day 14 after seeding.

Immunodetection. Immunofluorescence assay, Western blotting, and immunohistochemistry were as previously described $(5,30)$. A rat monoclonal antibody to LANA (Abcam), mouse monoclonal antibodies to ORF65 (5), vimentin (BioCare Medical), and Thy1.1 (Santa Cruz Biotechnology Inc.), and rabbit polyclonal antibodies to vWF (Thermo Fisher Scientific), PROX-1 (Covance), Ki67 (Abcam), and CD31 (Abcam) were used. Rabbit polyclonal antibodies to VE-cadherin, $\beta$-catenin, ICAM-1, VCAM-1, LYVE-1, VEGFR-1, and VEGFR-3 and goat polyclonal antibodies to podoplanin and CD34 were from Santa Cruz Biotechnology Inc.

Cell cycle and CFSE analysis and BrdU incorporation. Cell cycle was analyzed by propidium iodide staining. For CFSE assays, cells pulsed with $10 \mu \mathrm{M}$ CFSE were analyzed daily for relative intensities (Invitrogen). $\mathrm{BrdU}$ incorporation was determined by pulsing cells with $10 \mu \mathrm{M}$ BrdU for 4 hours, followed by staining with a mouse monoclonal antibody to BrdU (Invitrogen).

Growth of tumors. Subcutaneous growth of tumors was as previously described (29). Tumor volumes were measured twice a week using $0.2 \mathrm{~cm}^{3}$ as a threshold. Tumor analyses were performed at 20 weeks following inoculation or when the volume reached $1 \mathrm{~cm}^{3}$. Tumor images were taken at the end of study. Fluorescence images were taken with an Image Station 2000R System (Kodak).

Statistics. Data are shown as mean \pm SD. The 1-tailed Student's test was used to compare data between experimental groups. Statistical significance was assumed at $P$ values less than 0.05 .

Study approval. The animal protocol was approved by the Institutional Animal Care and Use Committee of the University of Texas Health Science Center at San Antonio (Animal Welfare Assurance no. A3345-01).

\section{Acknowledgments}

This work was supported by grants from the NIH (CA096512, CA124332, and CA132637) to S.-J. Gao.

Received for publication April 15, 2011, and accepted in revised form December 14, 2011.

Address correspondence to: Shou-Jiang Gao, University of Southern California, Keck School of Medicine, Los Angeles, California 90033, USA. Phone: 323.442.8028; Fax: 323.442.1721; E-mail: shoujiag@usc.edu.

1. Parkin DM. The global health burden of infectionassociated cancers in the year 2002. IntJ Cancer. 2006; 118(12):3030-3044.

2. Ganem D. KSHV and the pathogenesis of Kaposi sarcoma: listening to human biology and medicine. J Clin Invest. 2010;120(4):939-949.

3. Ciufo DM, et al. Spindle cell conversion by Kaposi's sarcoma-associated herpesvirus: formation of colonies and plaques with mixed lytic and latent gene expression in infected primary dermal microvascular endothelial cell cultures. J Virol. 2001; 75(12):5614-5626

4. Flore O, Rafii S, Ely S, O’Leary JJ, Hyjek EM, Cesarman E. Transformation of primary human endothelial cells by Kaposi's sarcoma-associated herpesvirus. Nature. 1998;394(6693):588-592.

5. Gao SJ, Deng JH, Zhou FC. Productive lytic replication of a recombinant Kaposi's sarcoma-associated herpesvirus in efficient primary infection of primary human endothelial cells. J Virol. 2003;
77(18):9738-9749

6. Moses AV, et al. Long-term infection and transformation of dermal microvascular endothelial cells by human herpesvirus 8. JVirol. 1999;73(8):6892-6902.

7. Carroll PA, Brazeau E, Lagunoff M. Kaposi's sarcoma-associated herpesvirus infection of blood endothelial cells induces lymphatic differentiation. Virology. 2004;328(1):7-18.

8. Hong YK, et al. Lymphatic reprogramming of blood vascular endothelium by Kaposi sarcoma-associated herpesvirus. Nat Genet. 2004;36(7):683-685.

9. Lagunoff $M$, et al. De novo infection and serial transmission of Kaposi's sarcoma-associated herpesvirus in cultured endothelial cells. J Virol. 2002; 76(5):2440-2448.

10. Wang HW, et al. Kaposi sarcoma herpesvirusinduced cellular reprogramming contributes to the lymphatic endothelial gene expression in Kaposi sarcoma. Nat Genet. 2004;36(7):687-693.

11. An FQ, et al. Long-term-infected telomerase- immortalized endothelial cells: a model for Kaposi's sarcoma-associated herpesvirus latency in vitro and in vivo. J Virol. 2006;80(10):4833-4846

12. Massarelli G, Scott CA, Ibba M, Tanda F, Cossu A. Immunocytochemical profile of Kaposi's sarcoma cells: their reactivity to a panel of antibodies directed against different tissue cell markers. Appl Pathol. 1989;7(1):34-41.

13. Browning PJ, et al. Identification and culture of Kaposi's sarcoma-like spindle cells from the peripheral blood of human immunodeficiency virus-1-infected individuals and normal controls. Blood. 1994;84(8):2711-2720.

14. Parsons CH, Szomju B, Kedes DH. Susceptibility of human fetal mesenchymal stem cells to Kaposi sarcoma-associated herpesvirus. Blood. 2004; 104(9):2736-2738.

15. Wu W, et al. KSHV/HHV-8 infection of human hematopoietic progenitor (CD34+) cells: persistence of infection during hematopoiesis in vitro 
and in vivo. Blood. 2006;108(1):141-151.

16. Mutlu $\mathrm{AD}$, et al. In vivo-restricted and reversible malignancy induced by human herpesvirus- 8 KSHV: a cell and animal model of virally induced Kaposi's sarcoma. Cancer Cell. 2007;11(3):245-258.

17. Zhou FC, et al. Efficient infection by a recombinant Kaposi's sarcoma-associated herpesvirus cloned in a bacterial artificial chromosome: application for genetic analysis. J Virol. 2002;76(12):6185-6196.

18. Aluigi MG, et al. KSHV sequences in biopsies and cultured spindle cells of epidemic, iatrogenic and Mediterranean forms of Kaposi's sarcoma. Res Virol. 1996; 147(5):267-275.

19. Lebbe C, et al. Characterization of in vitro culture of HIV-negative Kaposi's sarcoma-derived cells. In vitro responses to alfa interferon. Arch Dermatol Res. 1997;289(7):421-428.

20. Nakamura S, et al. Kaposi's sarcoma cells: long-term culture with growth factor from ret- rovirus-infected CD4+ T cells. Science. 1988; 242(4877):426-430

21. Salahuddin SZ, et al. Angiogenic properties of Kaposi's sarcoma-derived cells after long-term culture in vitro. Science. 1988;242(4877):430-433.

22. Fina $L$, et al. Expression of the CD34 gene in vascular endothelial cells. Blood. 1990;75(12):2417-2426.

23. Pont S. Thy-1: a lymphoid cell subset marker capable of delivering an activation signal to mouse $\mathrm{T}$ lymphocytes. Biochimie. 1987;69(4):315-320.

24. Abeysinghe HR, et al. THY1 expression is associated with tumor suppression of human ovarian cancer. Cancer Genet Cytogenet. 2003;143(2):125-132.

25. Nishinakamura R, Uchiyama Y, Sakaguchi M, Fujimura S. Nephron progenitors in the metanephric mesenchyme. Pediatr Nephrol. 2011; 26(9):1463-1467.

26. Usui J, Yamada R, Kanemoto K, Koyama A, Nagata $\mathrm{M}$. Murine metanephric mesenchyme possesses characteristics of vascular endothelial cells in vitro. Nephron Exp Nephrol. 2006;102(3-4):e93-e98.

27. Arar M, Xu YC, Elshihabi I, Barnes JL, Choudhury GG, Abboud HE. Platelet-derived growth factor receptor beta regulates migration and DNA synthesis in metanephric mesenchymal cells. J Biol Chem. 2000;275(13):9527-9533.

28. Wagner B, et al. Mitogenic signaling via platelet-derived growth factor beta in metanephric mesenchymal cells. J Am Soc Nephrol. 2007; 18(11):2903-2911.

29. Gao SJ, Boshoff C, Jayachandra S, Weiss RA, Chang Y, Moore PS. KSHV ORF K9 (vIRF) is an oncogene which inhibits the interferon signaling pathway. Oncogene. 1997;15(16):1979-1985.

30. Ye FC, et al. Kaposi's sarcoma-associated herpesvirus promotes angiogenesis by inducing angiopoietin-2 expression via AP-1 and Ets1. J Virol. 2007; 81(8):3980-3991. 